\title{
Anomalous magnetic anisotropy and magnetic nanostructure in pure Fe induced by high-pressure torsion straining
}

\author{
Y. Oba,${ }^{1, *}$ N. Adachi $\odot,{ }^{2}$ Y. Todaka $\odot,{ }^{2}$ E. P. Gilbert,${ }^{3}$ and H. Mamiya ${ }^{4}$ \\ ${ }^{1}$ Materials Sciences Research Center, Japan Atomic Energy Agency, 2-4 Shirakata, Tokai, Ibaraki 319-1195, Japan \\ ${ }^{2}$ Department of Mechanical Engineering, Toyohashi University of Technology, 1-1 Hibarigaoka, Tempaku, Toyohashi, Aichi 441-8580, Japan \\ ${ }^{3}$ Australian Centre for Neutron Scattering, Australian Nuclear Science and Technology Organization, \\ Locked Bag 2001, Kirrawee DC, NSW 2232, Australia \\ ${ }^{4}$ Quantum Beam Unit, National Institute for Materials Science, 1-2-1 Sengen, Tsukuba, Ibaraki 305-0047, Japan
}

(Received 4 November 2019; revised 23 July 2020; accepted 25 August 2020; published 23 September 2020)

\begin{abstract}
The formation of nanosized spin misalignment can be observed in pure Fe processed via high-pressure torsion (HPT) straining. The magnetic field dependence of the small-angle neutron-scattering profiles indicates that spin misalignment is conserved in magnetic fields up to $10 \mathrm{~T}$. This result demonstrates that HPT straining provides anomalous magnetic anisotropy in pure Fe due to the high densities of the grain boundaries and lattice defects.
\end{abstract}

DOI: 10.1103/PhysRevResearch.2.033473

\section{INTRODUCTION}

High-pressure torsion (HPT) is a renowned severe plastic deformation technique in metallurgy since it dramatically changes mechanical properties such as the tensile strength of structural materials [1-5]. Further, the effects of HPT on various metals and alloys have been extensively investigated for the development of new high-strength materials. Both the high density of crystal defects and the large fraction of grain boundaries, produced via HPT, function as pinning points to prevent dislocation motion, leading to an enhancement in the strength of metals and alloys.

The HPT process has also attracted attention in the field of magnetic materials [1,4,6-13]. The characteristic nanostructures produced using the HPT process considerably modifies the crystal growth kinetics [8,9]. Moreover, they can affect the magnetic properties owing to the pinning of magnetic domain walls and variation in exchange coupling between the grains [14-16]. Several researchers have reported an increase in the coercivity of magnetic materials via HPT straining [10,11]. These results provide a novel perspective that the HPT process can control magnetic anisotropy-one of the most important properties for spintronic devices-as well as broadening potential applications of both hard and soft magnetic materials [17-19]. In addition, magnetic anisotropy plays a key role in theories of magnetism, such as superparamagnetism [20,21].

In this study anomalous magnetic anisotropy produced via HPT straining in pure Fe is reported using small-angle neutron scattering (SANS), which is an excellent experimental technique for characterizing the nanostructure. SANS also enables magnetic structures to be probed due to the interaction

\footnotetext{
*Corresponding author: ohba.yojiro@ jaea.go.jp

Published by the American Physical Society under the terms of the Creative Commons Attribution 4.0 International license. Further distribution of this work must maintain attribution to the author(s) and the published article's title, journal citation, and DOI.
}

between the intrinsic spin of the neutron and the magnetic moments of the samples [22-26].

\section{EXPERIMENT}

Pure iron $(\mathrm{Fe})$ was selected as the sample for conducting this study. In terms of mass, the impurities were $\mathrm{C}, \mathrm{N}, \mathrm{O}$, and Al with values of $11,8,14$, and $300 \mathrm{ppm}$, respectively. The Fe sheet was cut into disks with a diameter of $20 \mathrm{~mm}$ and thickness of $0.85 \mathrm{~mm}$. The HPT process has been described in detail in a previous study [3]. Briefly, the Fe disk was held between two anvils and torsion strained at a pressure of $5 \mathrm{GPa}$, ten times at room temperature, with a rotation speed of $0.2 \mathrm{rpm}$. After the HPT process was completed, the samples were mechanically polished for adjusting the sample shape and thickness. Subsequently, the microstructure was observed using a scanning electron microscope (SEM) and analyzed based on electron backscatter diffraction (EBSD). Magnetic data were obtained using a commercial superconducting quantum interference device (SQUID) magnetometer (Quantum Design MPMS-5) with a magnetic field applied parallel to the disk.

SANS experiments were performed using the SANS instrument QUOKKA of the Australian Nuclear Science and Technology Organization (ANSTO) [27]. Four disks were stacked for measurements. The thickness of each disk was set to $0.5 \mathrm{~mm}$ by polishing. By comparing the scattering profiles of one disk with those of four stacked disks, it was confirmed that multiple scattering is negligible and does not affect the results (Fig. S1) [28]. A superconducting magnet was used to apply a magnetic field of up to $10 \mathrm{~T}$ to the samples parallel to the disks. Experiments were performed at equal source-to-sample and sample-to-detector distances of 20 and $8 \mathrm{~m}$ with source and sample aperture diameters of 50 and $15 \mathrm{~mm}$, respectively. A $q$ range from 0.03 to $1.0 \mathrm{~nm}^{-1}$ was explored, where $q$ denotes the magnitude of the scattering vector and is defined as $q=4 \pi \sin (\theta) / \lambda$, and where $\theta$ and $\lambda$ denote half the scattering angle and wavelength $(=0.5 \mathrm{~nm}$ with $10 \%$ resolution), respectively. The obtained scattering 


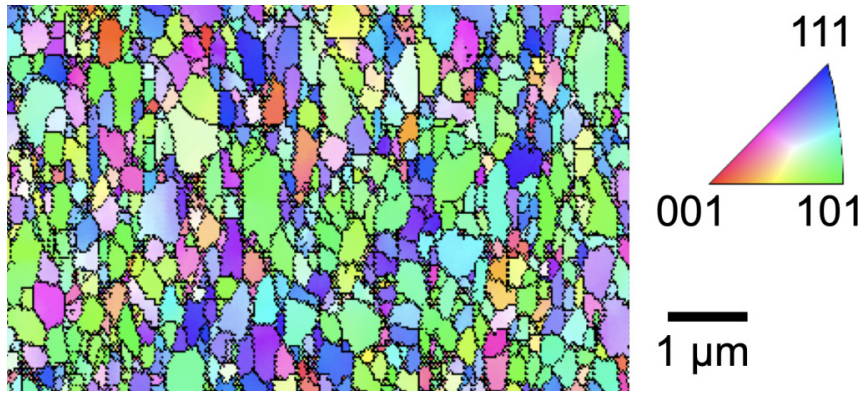

FIG. 1. IPF map of HPT-Fe obtained using EBSD.

patterns were radially averaged and corrected for background and transmission based on Refs. [27] and [29]. Details of the obtained scattering patterns are shown in the Supplemental Material (Figs. S2, S3, and S4) [28].

\section{RESULTS AND DISCUSSION}

Figure 1 shows the inverse pole figure (IPF) map of the HPT-strained Fe (HPT-Fe) obtained using EBSD. The average grain size is estimated to be $380 \mathrm{~nm}$, which is comparable with that described in a previous study and indicates that the crystal grains are remarkably refined through HPT straining [3]. The sample has minimal crystallographic anisotropy.

Figure 2 shows the magnetic field dependence of magnetization in HPT-Fe and nondeformed Fe. The coercivity increases from nearly 0 to approximately $2 \mathrm{mT}$ as a result of HPT straining. This may be attributed to the pinning of magnetic domain walls due to the high-density crystal defects and grain boundaries produced through HPT straining, as reported in previous studies [10,11]. The magnetization is rapidly saturated with a magnetic field lower than $0.5 \mathrm{~T}$

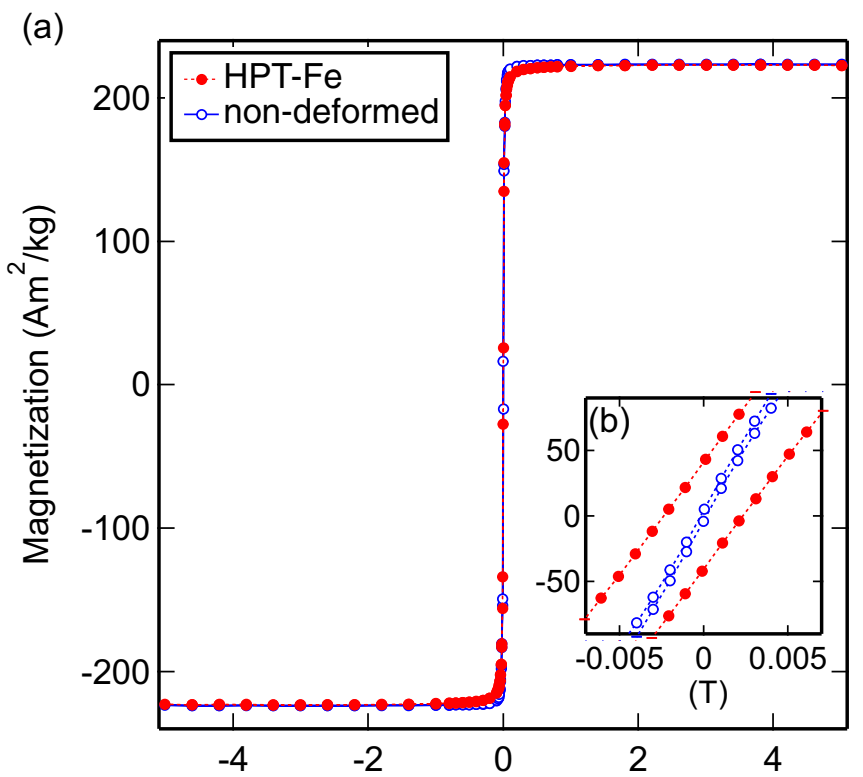

Magnetic field (T)

FIG. 2. (a) Hysteresis loops of HPT-Fe and nondeformed Fe. The inset (b) shows an enlarged view at around zero field.
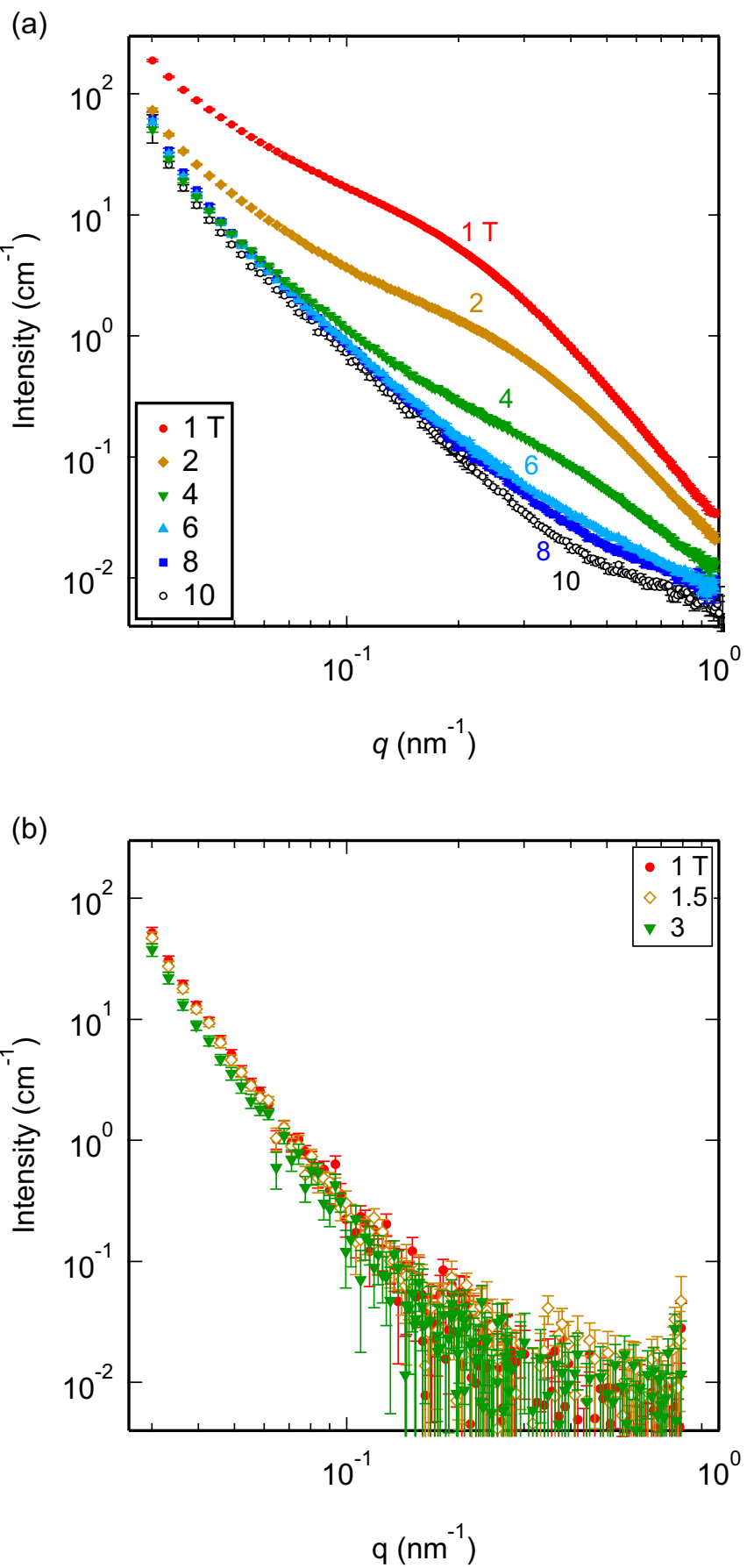

FIG. 3. SANS profiles of (a) HPT-Fe and (b) nondeformed Fe at several magnetic fields.

and exhibits no significant differences between HPT-Fe and nondeformed $\mathrm{Fe}$ at greater magnetic fields. The deviation of the magnetization in HPT-Fe from nondeformed Fe is below $1 \%$ at $1 \mathrm{~T}$ or higher fields (Fig. S5) [28]. Hence, the difference in saturation magnetization is negligible within error.

SANS profiles of HPT-Fe measured at magnetic fields ranging from 1 to $10 \mathrm{~T}$ are shown in Fig. 3(a). The profile at $1 \mathrm{~T}$ exhibits a shoulder, indicating the presence of a nanostructure in the sample [30], whereas nondeformed Fe shows no shoulder in SANS profiles and little magnetic 
field dependence [Fig. 3(b)]. The intensity of this shoulder monotonically decreases with increasing magnetic field and almost disappears at $10 \mathrm{~T}$. The observed dependence in the magnetic field is attributed to the magnetic scattering contribution, as the nuclear nanostructure is expected to be independent of the magnetic field. This result indicates that the magnetic nanostructure is formed in Fe via HPT straining.

The magnetization curve demonstrates that the magnetization of HPT-Fe is almost saturated below $1 \mathrm{~T}$, as is the case of the nondeformed $\mathrm{Fe}$, whereas the shoulder in the SANS profile remains up to $10 \mathrm{~T}$. This indicates that most of the magnetic moments in HPT-Fe are already aligned with the external magnetic field below $1 \mathrm{~T}$, which, again, is the same as that observed in the case of nondeformed Fe. Despite only a small fraction of the nanosized regions exhibiting a different magnetization state and contributing to the varying SANS intensity with applied field, SANS has the advantage of being sensitive to the low volume fraction of nanostructures; this is widely demonstrated in steel research, where SANS can detect nanosized precipitates with a volume fraction of less than $1 \%$ dispersed in a steel matrix [31-33].

The magnetic scattering contribution to the SANS intensity can arise from the difference in the direction and/or magnitude of the magnetization between the nanosized region and the matrix $[23,26]$. Based on conventional magnetic scattering theories [23,26,34-40], the magnetic field dependence of the SANS intensity is generated from the spin-misalignment scattering contribution caused by perturbing internal fields. Therefore the magnetic field dependence of the shoulder in the SANS profile indicates the occurrence of nanosized spin misalignment in a saturated matrix. The decrease of the shoulder up to $10 \mathrm{~T}$ indicates that the magnetic moments in the nanosized region become aligned with the external magnetic field in HPT-Fe. Almost no remaining shoulder at $10 \mathrm{~T}$ indicates that the scattering at $10 \mathrm{~T}$ corresponds mainly to both nuclear scattering and magnetic scattering generated by coarse microstructures such as grain boundaries and surface roughness in addition to possible remnants of spin-misalignment scattering.

Previous studies reported that spin misalignment can be formed based on the balance between the magnetic anisotropy and internal magnetic fields $[19,23,26]$. The internal field is the sum of the external and demagnetizing fields. However, the demagnetizing field should not exceed the magnitude of the magnetization (i.e., approximately $2.2 \mathrm{~T}$ for pure $\mathrm{Fe}$ with a demagnetizing factor of 1). Therefore the formation of the spin misalignment in HPT-Fe is attributed to the effects of the anomalous magnetic anisotropy field induced via HPT straining. Two possibilities can explain this anomalous field in the nanosized regions: (i) the local change in the anisotropy field compared to nondeformed $\mathrm{Fe}$ (of which magnetocrystalline anisotropy will inevitably play a role) or (ii) the magnetic anisotropy energy is lower than the thermal energy; this results in a thermal fluctuation of the magnetic moments and the disappearance of the time-averaged spontaneous magnetization. The latter resembles superparamagnetism observed in magnetic nanoparticles [20,21].

Guinier analysis was conducted to estimate the size of the regions influenced by spin misalignment (Fig. S6) [28].

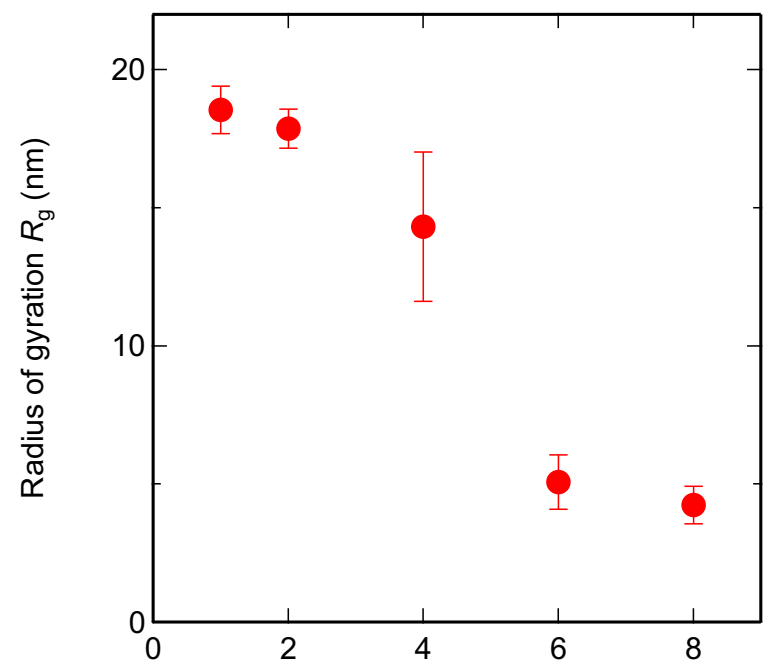

Magnetic field $(\mathrm{T})$

FIG. 4. Magnetic field dependence of radius of gyration $R_{\mathrm{g}}$.

This analytical technique does not require a detailed analytical model and is therefore suitable to characterize the present spin misalignment $[29,41,42]$. The SANS profile at $10 \mathrm{~T}$ with upturn with $q^{-4}$ power-law behavior was treated as "background" scattering from coarse structures and was subtracted from the remaining SANS profiles (Fig. S7) [28]. It is noted that a field of $10 \mathrm{~T}$ might be insufficient to completely suppress spin misalignment and reach ideal magnetic saturation; consequently, the spin-misalignment contribution is possibly underestimated. However, the approximate size estimation is still relevant to discuss the origin of the spin misalignment. To avoid possible effects of multiple scattering, Guinier analysis was performed in the range of $q>0.06 \mathrm{~nm}^{-1}$, where the deviation of scattering intensity between one disk and four disks is negligible within error (Fig. S1) [28]. Figure 4 presents the magnetic field dependence of the radii of gyration $R_{\mathrm{g}}$, which is a suitable measure of size of scattering objects and is numerically equal to $(3 / 5)^{1 / 2} R$ for monodisperse spherical nanoparticles with radius $R[29,41,42]$. The value of $R_{\mathrm{g}}$ decreases from 18.5 to $4 \mathrm{~nm}$ with increasing magnetic field. This result is significantly smaller than the average grain size that has been estimated based on the IPF map. Hence the spin misalignment does not correspond to the crystal grain.

In the case (i) described above, the spin-misalignment scattering contributions can be represented as the summation of scattering contributions $S_{\mathrm{H}}(\mathbf{q}) R_{\mathrm{H}}\left(q, \alpha, H_{i}\right)+$ $S_{\mathrm{M}}(\mathbf{q}) R_{\mathrm{M}}\left(q, \alpha, H_{i}\right)$ for the current scattering geometry within the approach-to-saturation regime [23,35,36]. Here, $S_{\mathrm{H}}(\mathbf{q}) R_{\mathrm{H}}\left(q, \alpha, H_{i}\right)$ and $S_{\mathrm{M}}(\mathbf{q}) R_{\mathrm{M}}\left(q, \alpha, H_{i}\right)$ are the scattering contributions due to perturbing magnetic anisotropy fields and magnetostatic fields, respectively. The parameters $\mathbf{q}, \alpha$, and $H_{i}$ denote the scattering vector, azimuthal angle between the scattering vector and the applied magnetic field direction, and magnitude of the internal field. The anisotropy-field scattering function $S_{\mathrm{H}}(\mathbf{q})$ is proportional to the Fourier coefficient of the magnetic anisotropy field, whereas the scattering 


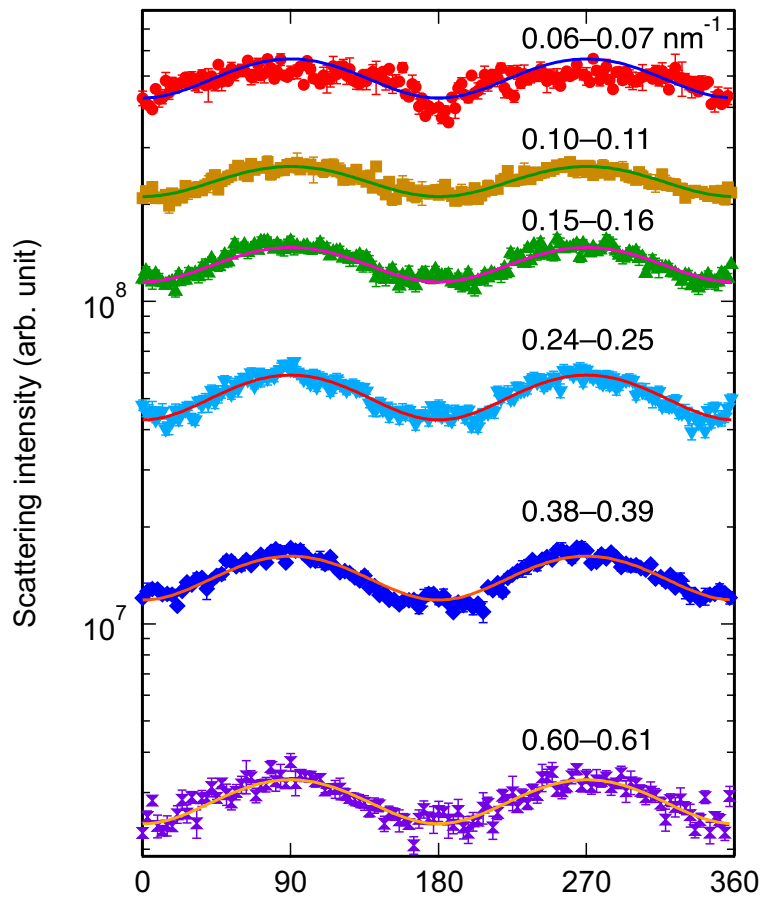

Azimuthal angle $\alpha$ (degree)

FIG. 5. Azimuthal angle dependence of scattering intensity in HPT-Fe at $1 \mathrm{~T}$. Plots are averaged in $q$ between 0.06 and $0.07,0.1$ and $0.11,0.15$ and $0.16,0.24$ and $0.25,0.38$ and 0.39 , and 0.6 and $0.61 \mathrm{~nm}^{-1}$. Solid curves are the fit results to $I_{0}+I_{\mathrm{s}} \sin ^{2} \alpha$, where $I_{0}$ and $I_{\mathrm{s}}$ are the constant fitting parameters corresponding to the intensity contributions with no azimuthal dependence and with $\sin ^{2} \alpha$ behavior, respectively.

function of the longitudinal magnetization $S_{\mathrm{M}}(\mathbf{q})$ reflects the spatial variations of the saturation magnetization. The functions $R_{\mathrm{H}}\left(q, \alpha, H_{i}\right)$ and $R_{\mathrm{M}}\left(q, \alpha, H_{i}\right)$ are the corresponding micromagnetic response functions, respectively. In isotropic magnetic materials, $R_{\mathrm{H}}\left(q, \alpha, H_{i}\right)$ and $R_{\mathrm{M}}\left(q, \alpha, H_{i}\right)$ bring peculiar azimuthal anisotropies to the two-dimensional scattering patterns. The contribution $S_{\mathrm{H}}(\mathbf{q}) R_{\mathrm{H}}\left(q, \alpha, H_{i}\right)$ is elongated parallel to the magnetic field, while $S_{\mathrm{M}}(\mathbf{q}) R_{\mathrm{M}}\left(q, \alpha, H_{i}\right)$ yields the so-called cloverleaf pattern.

In HPT-Fe, the orientation of the crystal grains is almost random, as shown by the IPF map (Fig. 1). Moreover, the shear strains introduced by HPT straining are along the circumferential direction in the disk plane [4]. Since the gauge volume of the SANS experiments was the center region of diameter of $15 \mathrm{~mm}$ and sample thickness, the remaining anisotropy in HPT-Fe must be averaged and can be regarded as random in the plane. However, the two-dimensional scattering patterns show neither the elongation parallel to the magnetic field nor cloverleaf-type azimuthal anisotropy, even after subtraction of the 10-T scattering pattern (Fig. S2) [28]. The azimuthal dependence of HPT-Fe at $1 \mathrm{~T}$ exhibits nearly $\sin ^{2} \alpha$ behavior, except in the lowest $q$ region (Fig. 5).

If the discrepancy in the azimuthal anisotropy is disregarded, the magnetic Guinier analysis can be applied, as described in the Supplemental Material (Fig. S9) [28,42,43].
This allows the evaluation of the radius of gyration for the magnetic anisotropy field $R_{\mathrm{GH}}$ and the exchange stiffness constant $A$. The value of $R_{\mathrm{GH}}{ }^{2}$ is estimated to be $0 \pm 15 \mathrm{~nm}^{2}$. If the square root of $15 \mathrm{~nm}^{2}$ is regarded as the experimental error, this suggests that the anomalous anisotropy field originates within a region smaller than $4 \mathrm{~nm}$. However, the estimated value of $A=35 \pm 7 \mathrm{pJ} / \mathrm{m}$ in HPT-Fe is significantly greater than that of $21-25 \mathrm{pJ} / \mathrm{m}$ in normal $\mathrm{Fe}[44,45]$. This contrasts with previous reports on nanocrystalline $\mathrm{Ni}$ and $\mathrm{Co}$, where the values of $A$ were consistent with normal $\mathrm{Ni}$ and $\mathrm{Co}$, respectively [34-36]. This indicates that further improvement of the analytical model is required to obtain reliable results about the spin misalignments in HPT-Fe.

For case (ii), there are several theoretical and experimental studies concerning the SANS from superparamagnetic nanoparticles [37-40,46] where such materials can exhibit two-dimensional scattering patterns with $\sin ^{2} \alpha$ behavior. This feature coincides with the results in HPT-Fe contrary to case (i) (Fig. 5). However, these previous studies have considered uniformly magnetized nanoparticles and have not provided the information about the behaviors of intraparticle magnetic domain structures. Furthermore, although they have claimed the domain structures expanded over several nanoparticles, the magnetic field dependences of domain sizes have not been formulated in those studies. Hence, this analysis method is also not applicable to HPT-Fe. To analyze the change in the magnetic domain size within nanosized regions, further improvement is necessary using micromagnetics. This method continues to progress with experimental, analytical, and micromagnetic-computational approaches [47-49]; the analytical solution for magnetic-field-dependent SANS intensity is only provided for a few special cases such as magnetic vortices in submicron-sized soft magnetic disks [48].

The discrepancies in both cases (i) and (ii) mean that the nanosized spin misalignments in HPT-Fe are probably different from the phenomena represented in conventional magnetic SANS theories. In the following, the possible origin of the nanosized spin misalignments is discussed in the context of the current SANS results. The magnetization curves indicate that the spin misalignment represents a small volume fraction of the sample, while the SANS profiles indicate that the size of the spin-misaligned region is smaller than the average grain size. Hence it is reasonable to conclude that the spin misalignment is probably generated only in a limited region such as around the high-density crystal defects and grain boundaries produced via HPT straining. The change in crystallographic symmetry can affect the magnetic anisotropy in such regions. For instance, several studies have reported that an increase of tetragonality in Fe, supported by interstitial nitrogen atoms, can increase the magnetic anisotropy energy [50-53]. Theoretical studies explain that the tetragonality in Fe stabilizes orbital magnetic moments and enhances the magnetic anisotropy via spin-orbit coupling [53,54]. Although voids and impurity nanoparticles may be regarded as the pinning points with respect to the motion of the magnetic domain walls [55], no shoulder can be observed in the case of residual scattering at $10 \mathrm{~T}$, indicating that HPT-Fe is likely to contain few such nanostructures. 


\section{CONCLUSION}

The magnetic field dependence of SANS confirms the formation of nanosized spin misalignment in $\mathrm{Fe}$ via HPT straining. The spin misalignment can be observed in magnetic fields up to $10 \mathrm{~T}$. The results obtained in this study indicate that anomalous magnetic anisotropy is induced in HPT-Fe. The spin misalignment is inconsistent with crystal grain size and is probably formed only around the high-density crystal defects and grain boundaries produced via HPT straining.

\section{ACKNOWLEDGMENTS}

The authors are grateful to the ANSTO Sample Environment group, particularly Dr. Norman Booth, as well as Mr. K. Yamamoto and Dr. N. Sato, for their help in this experiment. The authors would also like to thank Dr. K. Hiroi and
Professor A. Michels for useful discussions. We acknowledge the support of the Australian Centre for Neutron Scattering at ANSTO in providing the neutron research facilities used in this work as part of Proposal No. P5264. Some of this work was supported by KAKENHI Grant No. 19K05102, Japan Science and Technology Agency (JST), under Collaborative Research Based on Industrial Demand "Heterogeneous Structure Control: Towards Innovative Development of Metallic Structural Materials" (Grant No. JPMJSK1511), and the Research Group "Quantum-Beam Analysis of Microstructures and Properties of Steels" of the Iron and Steel Institute of Japan (ISIJ). Travel expenses for the SANS experiments were supported by the General User Program for Neutron Scattering Experiments, Institute for Solid State Physics, The University of Tokyo (Proposals No. 15563, No. 16554, and No. 16566), at JRR-3, Japan Atomic Energy Agency, Tokai, Japan.
[1] R. Z. Valiev, R. K. Islamgaliev, and I. V. Alexandrov, Prog. Mater. Sci. 45, 103 (2000).

[2] M. A. Meyers, A. Mishra, and D. J. Benson, Prog. Mater. Sci. 51, 427 (2006).

[3] Y. Todaka, M. Umemoto, J. Yin, Z. Liu, and K. Tsuchiya, Mater. Sci. Eng. A 462, 264 (2007).

[4] A. P. Zhilyaev and T. G. Langdon, Prog. Mater. Sci. 53, 893 (2008).

[5] K. Edalati and Z. Horita, Mater. Sci. Eng. A 652, 325 (2016).

[6] K. Y. Mulyukov, G. F. Korznikova, M. B. Sagdatkireyeva, V. N. Timofeyev, and R. Z. Valiev, J. Magn. Magn. Mater. 110, 73 (1992).

[7] K.Y. Mulyukov, G. F. Korznikova, and S. A. Nikitin, J. Magn. Magn. Mater. 153, 241 (1996).

[8] W. Li, L. Li, Y. Nan, Z. Xu, X. Zhang, A. G. Popov, D. V. Gunderov, and V. V. Stolyarov, J. Appl. Phys. 104, 023912 (2008).

[9] S. Lee, K. Edalati, H. Iwaoka, Z. Horita, T. Ohtsuki, and T. Ohkochi, Philos. Mag. Lett. 94, 639 (2014).

[10] S. G. Protasova, B. B. Straumal, S. V. Dobatkin, D. Goll, G. Schütz, B. Baretzky, A. A. Mazilkin, and A. N. Nekrasov, J. Mater. Sci. 43, 3775 (2008).

[11] S. Scheriau, M. Kriegisch, S. Kleber, N. Mehboob, R. Grössinger, and R. Pippan, J. Magn. Magn. Mater. 322, 2984 (2010).

[12] G. F. Korznikova, J. Microsc. 239, 239 (2010).

[13] A. Hosokawa, H. Ohtsuka, T. Li, S. Ii, and K. Tsuchiya, Mater. Trans. 55, 1286 (2014).

[14] J. B. Goodenough, Phys. Rev. 95, 917 (1954).

[15] J. Degauque, B. Astie, J. L. Porteseil, and R. Vergne, J. Magn. Magn. Mater. 26, 261 (1982).

[16] G. Herzer, J. Magn. Magn. Mater. 294, 99 (2005).

[17] O. Gutfleisch, M. A. Willard, E. Brück, C. H. Chen, S. G. Sankar, and J. Ping Liu, Adv. Mater. 23, 821 (2011).

[18] S. Sugimoto, J. Phys. D: Appl. Phys. 44, 064001 (2011).

[19] A. Brataas, A. D. Kent, and H. Ohno, Nat. Mater. 11, 372 (2012).

[20] C. P. Bean and J. D. Livingston, J. Appl. Phys. 30, S120 (1959).

[21] W. F. Brown, Phys. Rev. 130, 1677 (1963).
[22] A. Wiedenmann, J. Appl. Crystallogr. 33, 428 (2000).

[23] A. Michels, J. Phys.: Condens. Mater. 26, 383201 (2014).

[24] M. R. Fitzsimmons, S. D. Bader, J. A. Borchers, G. P. Felcher, J. K. Furdyna, A. Hoffmann, J. B. Kortright, I. K. Schuller, T. C. Schulthess, S. K. Sinha, M. F. Toney, D. Weller, and S. Wolf, J. Magn. Magn. Mater. 271, 103 (2004).

[25] Y. Oba, S. Morooka, K. Ohishi, N. Sato, R. Inoue, N. Adachi, J. Suzuki, T. Tsuchiyama, E. P. Gilbert, and M. Sugiyama, J. Appl. Crystallogr. 49, 1659 (2016).

[26] S. Mühlbauer, D. Honecker, É. A. Périgo, F. Bergner, S. Disch, A. Heinemann, S. Erokhin, D. Berkov, C. Leighton, M. R. Eskildsen, and A. Michels, Rev. Mod. Phys. 91, 015004 (2019).

[27] K. Wood, J. P. Mata, C. J. Garvey, C.-M. Wu, W. A. Hamilton, P. Abbeywick, D. Bartlett, F. Bartsch, P. Baxter, N. Booth, W. Brown, J. Christoforidis, D. Clowes, T. d'Adam, F. Darmann, M. Deura, S. Harrison, N. Hauser, G. Horton, D. Federici et al., J. Appl. Crystallogr. 51, 294 (2018).

[28] See Supplemental Material at http://link.aps.org/supplemental/ 10.1103/PhysRevResearch.2.033473 for details of the scattering patterns, deviation of the magnetization, and Guinier plots of HPT-Fe.

[29] S. R. Kline, J. Appl. Crystallogr. 39, 895 (2006).

[30] A. Guinier and G. Fournet, Small-Angle Scattering of X-rays (Wiley, New York, 1955).

[31] M. Ohnuma, J. Suzuki, S. Ohtsuka, S.-W. Kim, T. Kaito, M. Inoue, and H. Kitazawa, Acta Mater. 57, 5571 (2009).

[32] A. Deschamps, F. Danoix, F. De Geuser, T. Epicier, H. Leitner, and M. Perez, Mater. Lett. 65, 2265 (2011).

[33] Y. Oba, S. Koppoju, M. Ohnuma, T. Murakami, H. Hatano, K. Sasakawa, A. Kitahara, and J. Suzuki, ISIJ Int. 51, 1852 (2011).

[34] A. Michels, J. Weissmüller, A. Wiedenmann, and J. G. Barker, J. Appl. Phys. 87, 5953 (2000).

[35] J. Weissmüller, A. Michels, J. G. Barker, A. Wiedenmann, U. Erb, and R. D. Shull, Phys. Rev. B 63, 214414 (2001).

[36] D. Honecker, F. Döbrich, C. D. Dewhurst, A. Wiedenmann, and A. Michels, J. Phys.: Condens. Mater. 23, 016003 (2011).

[37] R. Pynn, J. B. Hayter, and S. W. Charles, Phys. Rev. Lett. 51, 710 (1983). 
[38] J. Kohlbrecher, A. Wiedenmann, and H. Wollenberger, Z. Phys. B 104, 1 (1997).

[39] A. Wiedenmann, Physica B 297, 226 (2001).

[40] J. F. Löffler, H. B. Braun, W. Wagner, G. Kostorz, and A. Wiedenmann, Phys. Rev. B 71, 134410 (2005).

[41] L. A. Feigin and D. I. Svergun, Structure Analysis by SmallAngle X-ray and Neutron Scattering (Plenum Press, New York, 1987).

[42] A. Michels, A. Malyeyev, I. Titov, D. Honecker, R. Cubitt, E. Blackburn, and K. Suzuki, IUCrJ 7, 136 (2020).

[43] D.-X. Chen, J. A. Bryg, and R. B. Goldfarb, IEEE Trans. Magn. 27, 3601 (1991).

[44] C. Antoniak, J. Lindner, K. Fauth, J.-U. Thiele, J. Minár, S. Mankovsky, H. Ebert, H. Wende, and M. Farle, Phys. Rev. B 82, 064403 (2010).

[45] K. L. Metlov, K. Suzuki, D. Honecker, and A. Michels, Phys. Rev. B 101, 214410 (2020).

[46] R. C. Woodward, J. Heeris, T. G. St. Pierre, M. Saunders, E. P. Gilbert, M. Rutnakornpituk, Q. Zhang, and J. S. Riffle, J. Appl. Cryst. 40, s495 (2007).
[47] A. Günther, J.-P. Bick, P. Szary, D. Honecker, C. D. Dewhurst, U. Keiderling, A. V. Feoktystov, A. Tschöpe, R. Birringer, and A. Michels, J. Appl. Cryst. 47, 992 (2014).

[48] K. L. Metlov and A. Michels, Sci. Rep. 6, 25055 (2016).

[49] L. G. Vivas, R. Yanes, and A. Michels, Sci. Rep. 7, 13060 (2017).

[50] M. Takahashi, Y. Takahashi, and H. Shoji, IEEE Trans. Magn. 37, 2179 (2001).

[51] N. Ji, M. S. Osofsky, V. Lauter, L. F. Allard, X. Li, K. L. Jensen, H. Ambaye, E. Lara-Curzio, and J.-P. Wang, Phys. Rev. B 84, 245310 (2011).

[52] I. Dirba, P. Komissinskiy, O. Gutfleisch, and L. Alff, J. Appl. Phys. 117, 173911 (2015).

[53] H. Zhang, I. Dirba, T. Helbig, L. Alff, and O. Gutfleisch, APL Mater. 4, 116104 (2016).

[54] T. Burkert, O. Eriksson, P. James, S. I. Simak, B. Johansson, and L. Nordström, Phys. Rev. B 69, 104426 (2004).

[55] K. Xu, D. K. Schreiber, Y. Li, B. R. Johnson, and J. McCloy, AIP Adv. 7, 056806 (2017). 